\title{
Legislative metadata based on semantic formal models
}

\section{Valentina Bartalesi Lenzi}

CELCT, Center for the Evaluation of Language and Communication Technologies

Via Sommarive 18, 38100 Povo, Italy

E-mail: bartalesi@celct.it

\section{Carlo Biagioli}

ITTIG, Institute of Legal Information Theory and Techniques

Italian National Research Council

Via dei Barucci 20, 50127 Firenze, Italy

E-mail: biagioli@ittig.cnr.it

\section{Amedeo Cappelli}

ISTI, Institute of Information Science and Technologies,

Italian National Research Council

Via Moruzzi 1, 56100 Pisa, Italy

E-mail: amedeo.cappelli@isti.cnr.it

CELCT, Center for the Evaluation of Language and Communication Technologies Via Sommarive 18, 38100 Povo, Italy

E-mail: cappelli@celct.it

\section{Rachele Sprugnoli}

CELCT, Center for the Evaluation of Language and Communication Technologies Via Sommarive 18, 38100 Povo, Italy

E-mail: sprugnoli@celct.it

\section{Fabrizio Turchi}

ITTIG, Institute of Legal Information Theory and Techniques

Italian National Research Council

Via dei Barucci 20, 50127 Firenze, Italy

E-mail: turchi@ittig.cnr.it

${ }^{*}$ Corresponding author

\begin{abstract}
The Law Making Environment system for planning, drafting and managing legislative sources is made up of editing and search support tools.

In order to handle the semantics of legislative sources, two interacting models have been developed: a rule model and a lightweight ontology. Semantic draft and search support tools are based on both models, in a way that makes their interaction possible. A rule theory has been developed, which can describe the illocutionary profile of legislative texts through metadata. As far as concepts are concerned, a conceptual dictionary has also been created. Through the search tool, called LMEmetaSearch, the user is enabled, for instance, to search for obligations related to particular actions or subjects, described in the query through the domain ontology. The LMEmetaSearch shows the related rules and additionally, if requested, finds implicit information providing the user with a wide account on the subject and playing to some extent the role of an advice system.
\end{abstract}

Keywords: legal models, legal ontologies, legal information, legislative meta searching, legislative meta drafting.

Biographical notes: Valentina Bartalesi Lenzi graduated in Humanities Computing at the University of Pisa in 2004, with a thesis on usability evaluation for museum web sites. She worked on the development of web access to cultural heritage and historical archives and she collaborated as web mistress with the University of Pisa. Since September 2005, she is a project consultant at CELCT working on semantic and speech annotation. 
Carlo Biagioli is a senior researcher at ITTIG-CNR, Florence. He works in the field of legal ICT, planning software systems to support legislative drafting. He studied formalisms to capture the normative profile semantics in the legislative texts, through provisions models and domain concepts ontologies. He was a member of the national working groups establishing standards for Italian legislation.

Amedeo Cappelli is a Research Director at the "Istituto di Scienza e Tecnologie dell'Informazione - A. Faedo" of the CNR in Pisa and the Scientific Director of CELCT. He has led many Italian and International research projects in the fields of Natural Language Processing and Artificial Intelligence. He currently teaches NLP and text analysis at the University of Pisa.

Rachele Sprugnoli graduated in Humanities Computing at the University of Pisa in 2004, with a thesis about the digitization of Renaissance Italian text. She did an internship at Philips in Eindhoven (The Netherlands) for the development of an Italian TTS system. Since June 2005, she is a project consultant at CELCT working on semantic and speech annotation.

Fabrizio Turchi is a senior technologist at ITTIG-CNR in Florence and received the degree in Mathematics from the University of Florence. His research activities include the application of information technologies to the legal domain, the theoretical topics linked to logic and logic programming applied to the law design, the use of XML language technologies for the construction of models of legal documents, and the design and development of web applications.

\section{INTRODUCTION}

The aim of the Law Making Environment (LME) project is the effective knowability of the legal order through Information Technology systems capable of reducing its complexity, giving legislators the opportunity to draft selfexplaining legislative texts, and giving citizens an easy access to them.

LME is a system of integrated tools, supporting the production and the management of legislative sources, and a direct search of relevant provisions. It is made of two modules, LMEmetaEdit and LMEmetaSearch.

LMEmetaEdit, which we developed in 2003 for the Italian Justice Minister, is an XML open source editor for the drafting support. It also allows the semantic mark-up of legal texts, during the drafting stage, or when the draft has already been drawn up, by inserting metadata according to a provision model and a concept model, developed as part of our project as well (Biagioli and Turchi 2005).

The Italian "Norme in Rete" (NiR) project has already promoted the standardisation of legislative sources and its annotation by means of metadata on acts and their contents, accepting our suggestion to add to the description of textual elements (partitions) the description of their regulative functions (Biagioli, 1997).

This paper is structured as follows. Section 2 introduces the functional structure of legislative texts and describes the formal provision theory developed in our project. Section 3 illustrates ontoPrivacy, a lightweight ontology developed to support the retrieval of information within the functional profile of legislative data. Sections 4 and 5 present the two modules integrated in the LME system, while Section 6 illustrates the methods on which the retrieval of correlated disposition is based. Section 7 reports on query expansions as performed in LMEmetaSearch giving several examples. Section 8 is devoted to provide a brief presentation of related works and, finally, some conclusions are drawn in Section 9.

\section{TOWARDS A FORMAL DISPOSITION THEORY}

\subsection{Functional structure of legislative texts}

The semantics of a text result from its atomic components (terms), its meaningful partitions (normative micro-acts), and the text as a whole (normative macro-act).

The functional elements are called dispositions, provisions, or rules, and are intended as typical legislative statements. The textual profile has given place to DTD-NiR, while the functional one has originated the analytical internal metadata.

Although no linear relationship can be found between surface and functional patterns in a legislative source, there is a consistent correspondence between text atomic items (paragraphs) and meaningful/functional units (dispositions or rules), intended as normative speech acts. Based on these findings, it can be reasonably argued that this is no casual correspondence: indeed each provision is, from a general standpoint, the meaning of each basic text unit (paragraph).

In Italian legal texts, each provision generally corresponds to a paragraph, but, in some cases, it may match with an internal partition (letter or number), an internal sentence, or a part of a sentence. Sometimes it is quite convenient, without losing effectiveness, to summarise provisions of the 
same type, contained in an article, in a single provision. Therefore, to avoid confusion, the identification of rules is based on the various illocutive profiles of underlying acts, rather than on fixed elements as textual partitions or sentences, as done in other projects (de Maat and Winkels, 2007).

The provisions were studied and modelled using a set of rules that expresses the basic reasons and meanings of legislating. Their contents are represented by modelling the language of the regulated domain, gathering relevant terms around concepts and inside classes, in accordance with the technique of building ontologies. While the model of rules is universal in the legislative domain, at least within a legal order, the modelling of concepts is relevant, at least in part, to the ruled domain.

Functional structure includes classes, types of dispositions and their elements (arguments). This classification comes from a cognitive analysis of legislative techniques and legislative practices, and also by taking into account theories on norms (Honoré 1977; Sartor 2006).

Dispositions are divided into two main classes, "metarules" and "rules". These are in turn separated into the two major categories, often referred to as "constitutive" and "regulatory" rules, according to the well-known distinction drawn by Rawls (1955): "justifying a practice and justifying a particular action falling under it", where practice stands for "any form of activity specified by a system of rules which defines offices, roles, moves, penalties, defences, and so on, and which gives the activity its structure". Classes can also be used in the semantic mark-up of complex partitions, having a more general meaning than dispositions.

Arguments are the main focus of rules and give them their logical structure. Their own arguments are always to be found in a provision of a certain type to be regarded as such (see, for instance, the definiendum for a definition, or the addressee for an obligation). Argument contents may be either single or multiple and appear in texts in various forms: explicit direct, explicit indirect (e.g. "the aforesaid"), explicit variable (e.g. "any entity interested"), and implicit (unknown).

In addition to the type of disposition, argument contents typically make up the information of section/part titles in Italian legislative sources: law makers use provision typology and keywords that summarise its contents to illustrate the ratio of the following partition.

To conclude, the legislative text has been deeply modelled as a set of rules, while each rule has been modelled as a framework made of several well-known internal components called arguments (slots). The contents of every argument are keywords that belong to a domain controlled vocabulary, organised into classes, as explained in section 3.

A double modelling approach has been in fact chosen. A disposition model expresses the pragmatic side that is how legislators organise and express rules about domain entities, while domain entities are classified and described ontologically. The double modelling of the pragmatic and semantic profiles of text meanings, is in tune with the (Breuker and Hoekstra 2004) statement "an ontology is in the first place a set of terminological definitions built around a taxonomic backbone, while a framework is an assembly of concepts or types of knowledge that reflect recurrent patterns of use".

\subsection{Formalization of Disposition Theory}

We cite from (Biagioli and Grossi, 2008):

"The formal provision theory developed in LME is a terminology (in the technical sense of description logic) for classifying fragments of legal texts. The domain of discourse is the set of textual fragments, that is to say, the formalization describes textual fragments and considers them as instances of metadata, i.e. the dispositions structured in the terminology.

The terminology of dispositions can be seen, therefore, as a theory of a possible set of metadata that can be used for the mark-up of legal texts. Metadata are isolated a priori and the logical relations holding between them are imposed axiomatically. As such, the terminology of disposition cannot be properly considered a theory of normative concepts ".

The formalization of the theory of provisions, amounts to a "logic of dispositions" and it can be viewed as an attempt towards a formal foundation of legistics (i.e. the set of techniques for legal drafting).

The description logic SHIF(D) has been used. Firstly, a sort of minimal theory of dispositions essentially corresponding to a taxonomy is provided. Stronger logical relations have been later on imposed in order to enable richer inference patterns and to better characterize the dispositions at issue.

In fact the LMEmetaSearch tool, while searching for rules in legislative sources, is also able to find, in a second step, implicit or complementary rules in comparison with the dispositions previously found. As a matter of fact, the system is able to run inferences, i.e. deductions from relations between rules, and deductions from relations between domain relevant concepts. We can distinguish the relationships that bind the rules into logical (implicit rules) and technical (complementary rules): implicit rules are deducted from deontic relations while complementary rules are identified from technical legislative (legistics) relations. These inferences avoid the introduction of unnecessary and too interpretative annotations (metadata) and they broaden the scope of explanatory replies, giving the system an ability to answer that is very similar to that of a consultant lawyer, as found comparing the responses to those of the web sites specialized in consultancy relating to the protection of personal data.

For their effective employment, arguments are to be synthesized and connected to structured dictionaries and terminological collections, as explained below. In practice, the rule model allows to highlight the terms of the dispositions that carry out a particular role (e.g. addressee), while the information on the meaning of the term (keyword) will be found in terminological collections. In other words, while in ontology-based searching systems all terms 
contained in the document are considered, in LMEmetaSearch only terms relevant according to the rule model (its arguments) are taken into account.

The LMEmetaSearch works with every classification of domain relevant keywords, from flat glossaries to deeply structured ontologies. The more complex is the description of domain entities, the easier it is to express complex content descriptions in the searching system queries.

On one hand, the role of deductions, based on the rule model, in identifying during the search phase norms that are implied or variously related is clear. On the other hand, the role of reasoning about concepts, classes and their relationships, for the purposes of identification of rules contents, is still under investigation, because a sufficiently broad archive of marked-up laws is required.

To test LMEmetaSearch, a domain ontology, concerning the personal data protection Italian law, has been developed.

Unfortunately, up till now, time and resource constraints have not permitted us to mark-up an entire legal domain. We are currently working on the semantic mark-up of a wider collection of Italian laws, to be able to investigate this point, which is still under examination, better.

\section{THE ROLE OF ONTOLOGY: ONTOPRIVACY}

Laws contain dispositions which deal with domain common sense entities (argument contents), but they do not provide any general information on them, except in the case of definitional provisions. The use of ontology allows the possibility to acquire such additional general information. Moreover ontology consents the user to obtain a normalized form of the terms by which entities are expressed, so that they can be indexed and used in the analytical metadata querying process of law document search and retrieval.

In recent years, there have been several initiatives for the development of legal ontologies in order to offer a solid support for the acquisition, sharing and reuse of legal knowledge. In particular, we have taken into consideration two core ontologies: 1) LKIF Core Ontology (Breuker et al., 2007), inspired by the LRI Core ontology effort and developed within the Estrella project; 2) Core Legal Ontology (Gangemi et al., 2005), organising juridical concepts in classes of entities that are specializations of the DOLCE + foundational ontology. At the same time, we have taken the Legal-RDF Vocabularies (http://www.legalrdf.org) as a reference. These Vocabularies have been developed by John McClure, Legal-RDF director, for the annotation of narrative legal documents in the Semantic Web perspective.

Our work focused on the study of the ontologies mentioned above in order to organise into classes the instances of a glossary of keywords extracted from the Italian Personal Data Protection Code (d.lgs. 196/2003). Therefore, ontoPrivacy can be considered a lightweight ontology consisting of a set of concepts and hierarchical relationships among them and not including axioms and constraints, peculiar to the heavyweight ontologies (Corcho et al., 2003).
The glossary on which our experiment is based, has been manually created and it is made up of terms specific to the Public Administration domain (e.g. atto amministrativo/administrative act), ruled domain words (e.g. dispositivo elettronico/electronic device), and generic words (e.g. parere/opinion). Synonyms, hypernyms and hyponyms are identified among such terms (e.g. administrative acts and judicial acts are kinds of acts).

Taking the relevant terms contained in the glossary as starting points, we have followed a bottom-up approach to create the ontology. Vocabulary and ontology are, in fact, closely tied with a two-way relation: a lexicon can be the basis for a well-built ontology, and an ontology can serve as the foundation for lexicon organisation (Hirst, 2003).

The classes in ontoPrivacy have been defined by analyzing the lexical entries of our glossary and the relations among them. ontoPrivacy has six main classes (see Figure 1) as described below.

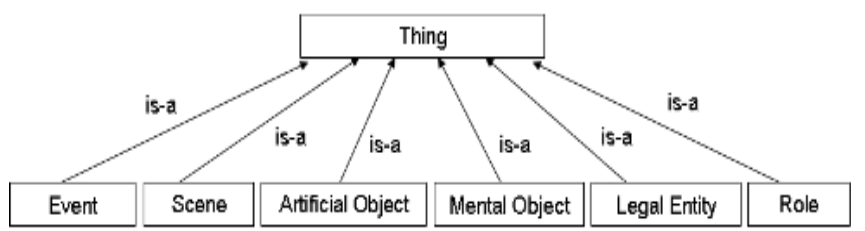

Figure 1: The upper part of ontoPrivacy

Event. An event is a situation that arises involving participants, and it can be described as a change of state. There are many Event subclasses. Each Event subclass is named by a noun-phrase (e.g. AgreementEvent). One or more keywords are assigned to each subclass: for example, decisione/decision and deliberazione/resolution are two instances of the DecisionEvent subclass.

Scene. The Scene class defines places and times in which an event occurs. A Place may be a boundary, a space, a structure, or a surface (e.g. paese extraeuropeo/non European state, Repubblica italiana/Italian Republic). A Temporal Expression (TE) can be a duration (e.g. tre anni/three years), a point (e.g. 17 Luglio 1999/July 17th 1999), or a set of times/ (e.g. ogni mese/every month). At present, there are no TEs in our glossary but we intend to use an automatic annotation tool to recognize and normalize a broad variety of TEs which can be found within an input text.

Artificial Object. Artificial Objects can be tools, machines, pieces of furniture, and other artefacts intentionally made to serve a given purpose (e.g. fibra ottica/optical mean, cavo/wire and dispositivo elettronico/electronic device).

Mental Object. A Mental Object is a non-physical object, a thing we mentally manipulate. This class is divided into two subclasses: Information Object (IO, e.g. sentenza/judgment) and Conceptualization (e.g. liceità/lawfulness). An IO is a reification of pure information as described in Shannon's communication theory (Shannon and Weaver, 1949). The IO subclass is further categorized in Juridical Information Object and General Information Object. 
Legal Entity. A legal entity is "any natural person or any legal person, provided that it has been established under Community law or the applicable national law and has been given legal personality or has the capacity, in its own name, to hold rights and obligations of all kinds, to conclude contracts and to be a party to legal proceedings" (1999/65/CE). The Legal Entity class is divided into two subclasses: Natural Person (e.g. cittadino/citizen) and Legal Person (e.g. sindacato/trade union). A legal entity always plays a role (i.e. a function performed by a certain entity) when it enters in relationships with other entities.

Role. A role is a function played by a legal entity. The Role class is divided into two subclasses: Social Role and Judiciary Role. The first class defines a role played by some agent in the context of social activities. It is divided into: (i) Organisation Role, a role that has a meaning in the context of an organisation: i.e. the role defines the "position" of a natural person within the structure of an organisation (e.g ministro/ministry); (ii) Legal Role, a role played by some legal person (e.g. comune/local municipality); (iii) Person Role, a role played by some natural person (e.g. lavoratore/worker). The Judiciary Role class defines the role played by some agent in the context of legal activities. This class has a Professional Judiciary Role as subclass. It identifies a legal profession of legal entities (e.g. pubblico ministero/public prosecutor). Figure 2 illustrates the Role class together with its dependent elements.

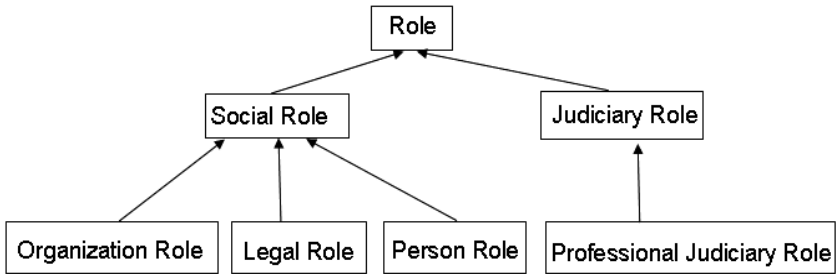

Figure 2: Role class together with its dependent elements

\section{LMEMETAEDIT}

The subject of this section is the Disposition-Argument model (D-A model) tested on a real legal domain. The user can describe the semantic contents of each source partition, first qualifying its disposition type through the provisions model, thus capturing the basic intention of the legislator. Secondly, the provision contents can be qualified through the available concept models, thus describing the content of each provision.

The semantic mark-up includes the ID partition, an unambiguous way to identify the partition of formal profile that represents the gateway between the syntactic and the semantic profile. For example, consider the following partition:

- $\quad$ article 22, paragraph 8: Data disclosing health may not be disseminated.

In the semantic mark-up process, the previous paragraph is seen as a prohibition, with the "partition" set as "art22-par8" and the "action" argument set as "disseminated" and the "object" argument set as "data health". The assignment of values to arguments relies on ontological classes, so that the available values belong to the appropriate ontological class. For example, a "counterpart/addressee" argument belongs to the "Legal Entity" class and the "action/activity" argument belongs to the "Event" class.

Mark-up strategies can range between two extreme ends. Documentalists can decide to mark only explicit entities, thus adopting a low degree of text interpretation. Otherwise, implicit information, detected thanks to an interpretative effort, can also be added to the marked-up entities. Obviously, the more complete is the mark-up, the wider the answer in the searching for norms will be.

The provisions belonging to the Metarules top class (Derogations and Modifications sub-classes), are dispositions acting on other provisions belonging to the Rule top class. They also belong to the nature (logical structure) of the acted provisions: a derogation to a permission rule is part of the whole permission, deriving from the connection of the derogated provision and the derogating one. Then the derogating provision has typical contents (arguments) of the derogated one. Therefore derogations can be doubly marked-up; as derogations and as provisions of the same type of the derogated provision.

\section{LMEMETASEARCH}

The semantic mark-up is the condition to use LMEmetaSearch, an open source tool to query the functional profile of legislative texts through the D-A model and relied on ontoPrivacy. This LME module allows the user to query the legislative information system on the basis of two modalities of reasoning: an interpretation based on provisions and their relations and/or on domain concepts and their relations.

The argument-setting value process can involve more than one single term, generally tied to the previous ones through Boolean operators. A further choice consists in using the linguistic expansions between terms, e.g. hyponym, hypernym and synonym relations.

After preparing the query, the system creates a new window, where the results, that satisfy the criteria set up in the query prepared, are shown. The result encompasses the following information:

- disposition type,

- formal partition (article and paragraph number);

- $\quad$ argument name;

- argument value;

- disposition text (on explicit user demand and extracted by a DOM parser that processes the legal act in XMLDTD-NiR format).

The smallest information shown is the single disposition with all its associated argument contents (metadata). 


\section{CORRELATED DISPOSITIONS}

In the legistic tradition provisions can be linked with references. This connection is referred to as syntactic relations (explicit links) for the rest of the paper. For instance, a derogation rule is usually linked to the derogated one to make this relation between the two provisions explicit.

The frequent lack of these explicit syntactic references makes the legal system harder to understand. The rule-based metadata can, to some extent, sidestep that difficulty, describing semantically, and thus allowing us to capture, some correlations between several disposition types, called semantic relations (conceptual links).

Semantic relations between dispositions have basically two foundations, logical (implicit rules) and technical (complementary rules), as explained in section 2.

Therefore, provisions can be retrieved, as correlated dispositions, in two distinct ways:

- relying on explicit reference, when possible;

- relying on the similar content of common arguments, between provisions of the first result set and provisions correlated according to the rule model.

In the implicit rules the renowned logic relations between deontic concepts are used as relations between the homonymous provisions. For example, when the user looks for the obligations of a given subject, the system also finds the implicit obligations that take the form of claims of the subject's counterpart. From these logic relations, implicit rules are inferred.

For what concerns the complementary rules, according to legistic knowledge and legislative drafting practices, legislators are used to establish explicit relations (syntactic relations) between the following different kinds of dispositions:

- Procedures - Obligations: procedural and substantial obligations on the same content, are often linked by internal references.

- Regulatives - Violations: a Violation normally makes references to a functionally related Regulative disposition.

- Rules - Metarules: any kind of Rule can be influenced and changed by Modifications and Derogations rules; in this case it is normally (but not always) referenced. Otherwise, semantic links are useful to solve that problem.

As explained in section 4, provisions that belong to the Derogations sub-class (Metarules class), can be marked-up as Metarules but as Rules too; this strategy allows us to capture and find derogation provisions also thanks to their semantic features. Therefore, derogative provisions can be retrieved, as correlated dispositions, in two distinct ways:

- relying on explicit reference, if a cross reference really exists;
- relying on the double mark-up, verifying the contents of common arguments, and the type of provisions of the first result set.

A legistic relation also relies on regulative and violation type of provisions: when they rule the same case in point, they have the same addressee and action argument contents. In the Italian legistic practice, regulative provisions and the connected violation dispositions are also usually (but not systematically) linked by a reference that takes the form of a numeric formula, or of a linguistic expression.

Related rules (implicit and complementary) can be found activating syntactic or semantic (legistic/logic) relations. Those two kinds of connections allow an expansion of the answer, adding, if requested, new related provisions and thus giving a more complete answer. The LMEmetaSearch allows the user to retrieve further dispositions, to be added to the result of a query, and to show the complete result as a union of the two sets: the first one extracted from the first query and the second one made by the correlated dispositions.

The aim is, in general, to turn searching into advice as much as possible, as a guide to the consultation of legislative sources.

\section{REASONING PROCESSES IN LMEMETASEARCH}

\subsection{Query expansions based on rule model}

Starting from the results page, the system allows the user to search for the correlated dispositions. As we said, there are two link forms:

- $\quad$ syntactic form;

- $\quad$ semantic form;

and two correlation types:

- implicit type;

- complementary type.

The syntactic correlation is based on the existence of an explicit normative reference among involved dispositions, while the semantic relation relies on the correspondence among common arguments contents (keywords) in pairs of related rules.

The semantic correlation can be seen as a dyadic or 2-fold relation: it puts together a number of pairs (Disposition, Argument), greater than or equal to 1 , so defined:

$$
\text { (Dx, Ax) - correlation-> (Dy, Ay) }
$$

where Dx is an $x$ disposition type and $\mathrm{A} x$ is an argument belonging to Dx, Dy is a $y$ disposition type and Ay is an argument belonging to Dy.

The pairs can be put in relation between themselves, that is, they can be part of the correlation, only if the associated arguments have at least one keyword in common. 
In other words, the arguments involved in the relation contain the same terms extracted from the controlled legal vocabulary.

We classify the correlation as follows:

- $\quad$ strong: if all the pairs meet the equivalence between the arguments and all the keywords involved are equal;

- weak: if not all the pairs meet the equivalence between the arguments or not all the keywords involved are equal.

Some examples of deduction of implicit and complementary rules, from the result of a query, are listed in next subsections in two steps, implicit rules (A) and complementary rules (B).

\subsubsection{A - Implicit rules}

Example 1. The user looks for the data controller obligations regarding the data subject (called, hereinafter, the first results set). The system retrieves the following paragraph:

- article 52, paragraph 4: "If judgments or other measures, or the corresponding head notes, bearing the notice as per paragraph 2 are disclosed also by third parties, the data subject's name and other identification data shall be omitted".

For what concerns the query expansion, if the user asks the system for the implicit obligations (i.e. claims semantically correlated to the dispositions of the first results set), he/she'll get the new following expanded results set:

- article 52, paragraph 4: "If judgments or other measures, ..."

- $\quad$ article 8, paragraph 2: "The rights referred to in Section 7 may not be exercised by making a request to the data controller or processor, or else by lodging a complaint in pursuance of Section 145, if the personal data are processed:

a) pursuant to the provisions of decree-law no. 143 of 3 May 1991, as converted, with amendments, into Act no. 197 of 5 July 1991 and subsequently amended, concerning money laundering;

b) pursuant to the provisions of decree-law no. 419 of 31 December 1991, as converted, with amendments, into Act no. 172 of 18 February 1992 and subsequently amended, concerning support for victims of extortion;

c) by parliamentary Inquiry Committees set up as per Article 82 of the Constitution;

d) by a public body other than a profit-seeking public body, where this is expressly required by a law for purposes exclusively related to currency and financial policy, the system of payments, control of brokers and credit and financial markets and protection of their stability;

e) in pursuance of Section 24(1), letter f), as regards the period during which performance of the investigations by defence counsel or establishment of the legal claim might be actually and concretely prejudiced;

f) by providers of publicly available electronic communications services in respect of incoming phone calls, unless this may be actually and concretely prejudicial to performance of the investigations by defence counsel as per Act no. 397 of 7 December 2000;

$\mathrm{g}$ ) for reasons of justice by judicial authorities at all levels and of all instances as well as by the Higher Council of the Judiciary or other self-regulatory bodies, or else by the Ministry of Justice;

h) in pursuance of Section 53, without prejudice to Act no. 121 of 1 April 1981".

The system is able to build the expanded result set, relying on the semantic profile of the dispositions involved. In fact, from the semantic point of view we have the following profile:

$$
\begin{array}{ll}
\text { obligation } & \left\{\begin{array}{l}
\text { partition }=\text { art52-par4 } \\
\text { addressee }=\text { data controller } \\
\text { counterpart }=\text { data subject } \\
\text { action }=\text { diffusion } \\
\text { object }=\text { personal data }
\end{array}\right. \\
\text { claim } & \left\{\begin{array}{l}
\text { partition }=\text { art8-par2 } \\
\text { addressee }=\text { data subject } \\
\text { counterpart }=\text { data controller }
\end{array}\right.
\end{array}
$$

In this case we have a weak semantic correlation because the arguments involved, (addressee, counterpart and action) don't fully satisfy the equivalence. In fact, the action argument in the claim disposition is not marked. It should be noted that in this case the strong correlation requires the equivalence of all the following three pairs:

1. (obligation, addressee $(k)) \longrightarrow($ claim, counterpart $(k))$

2. (obligation, counterpart $(k)) \longrightarrow($ claim, addressee $(k))$

3. (obligation, action $(k)) \longrightarrow($ claim, action $(k))$

The inference process we have just seen is crucial, because it allows the user to capture the different legislator styles. In fact, the legislator can express an obligation for an addressee as well as a claim for his counterpart.

\subsubsection{B - Complementary rules}

Example 1. The user looks for obligations about the assignment of personal data. The system retrieves, as the result set, the following paragraph:

- article 16, paragraph 1: "Should data processing be terminated, for whatever reason, the data shall be:

a) destroyed;

b) assigned to another data controller, provided they are intended for processing under terms that are compatible with the purposes for which the data have been collected;

c) kept for exclusively personal purposes, without being intended for systematic communication or dissemination; 
d) kept or assigned to another controller for historical, scientific or statistical purposes, in compliance with laws, regulations, Community legislation and the codes of conduct and professional practice adopted in pursuance of Section 12".

As for the query expansion, if users inquire for the violation dispositions syntactically correlated to the dispositions of the first results set, they will get the new expanded results set as follows:

- article 16, paragraph 1: "Should data processing be terminated, for whatever reason, ..."

- $\quad$ article 162, paragraph 1: "Assigning data in breach of Section 16, paragraph 1, letter b), and/or other provisions concerning the processing of personal data shall be punished by a fine consisting in payment of between five thousand and thirty thousand euro"

In this case the syntactic correlation relies on the explicit reference inserted by the legislator, i.e. Section 16, paragraph 1 . However, the rule model allows the user to retrieve the same violation, also through the semantic correlation. In fact, if the users inquire for the violation dispositions semantically correlated to the first results set, they will get the same expanded results set shown below:

- article 16, paragraph 1: "Should data processing be terminated, for whatever reason, ..."

- $\quad$ article 162, paragraph 1: "1. Assigning data in breach of Section 16, paragraph 1 , letter b), ..."

The algorithm used by the system to build the same expanded results set relies on the semantic profile of the dispositions involved. In fact, from the semantic point of view, we have the following profile:

$$
\begin{array}{ll}
\text { obligation } & \left\{\begin{array}{l}
\text { partition }=\text { art16-par } 1 \\
\text { action }=\text { assignment/destruction/retention } \\
\text { object }=\text { personal data }
\end{array}\right. \\
\text { violation } & \left\{\begin{array}{l}
\text { partition }=\text { art162-par1 } \\
\text { action }=\text { assignment } \\
\text { object }=\text { personal data } \\
\text { penalty = administrative sanction }
\end{array}\right.
\end{array}
$$

Also in this case, we have a weak semantic correlation because the arguments involved (action and object) don't satisfy all the arguments equivalence. In fact, only the "assignment" keyword is common in the action arguments. It should be noted that, in this example, the strong correlation requires the equivalence of the following two pairs:

1. (violation, action $(k)) \longrightarrow($ obligation, action $(k))$

2 . (violation, object $(k)) \longrightarrow($ obligation, object $(k))$

This inference process is important because if the legislator fails to explicitly indicate a normative reference, the user will be able to retrieve the correlated dispositions, even not explicitly linked, thanks to the rules model.
Example 2. The user looks for the data subjects rights about personal data communication. The system retrieves, as results set, the following paragraph:

- $\quad$ article 7, paragraph 1:“ A data subject shall have the right to obtain confirmation as to whether or not personal data concerning him exist, regardless of their being already recorded, and communication of such data in intelligible form"

For what concern the query expansion, if the users enquire for the semantic exceptions/derogations to the dispositions of the first results set, they will get the new following expanded results set:

- $\quad$ article 7, paragraph 1: "A data subject shall have the right to obtain confirmation ..."

- article 10, paragraph 5: "The right to obtain communication of the data in intelligible form does not apply to personal data concerning third parties, unless breaking down the processed data or eliminating certain items from the latter prevents the data subject's personal data from being understandable"

Once again the system is able to build the expanded result set, relying on the semantic profile of the dispositions involved. In fact, from the semantic point of view we have the following profile:

$$
\begin{aligned}
& \text { right }\left\{\begin{array}{l}
\text { partition }=\text { art7-par1 } \\
\text { addressee }=\text { data subject } \\
\text { activity }=\text { communication } \\
\text { object }=\text { personal data }
\end{array}\right. \\
& \text { exception }\left\{\begin{array}{l}
\text { partition }=\text { art10-par5 } \\
\text { norma }=\text { normative reference }
\end{array}\right. \\
& \text { right }^{t} \quad\left\{\begin{array}{l}
\text { partition }=\text { art10-par5 } \\
\text { addressee }=\text { data subject } \\
\text { activity }=\text { communication } \\
\text { object }=\text { personal data }
\end{array}\right.
\end{aligned}
$$

In this example, we have a strong semantic correlation because all the arguments involved (addressee, object and activity) meet all the arguments equivalences.

This query/answer represents a case of explicit reference (syntactic) expressed not in technical form with a reference, but with words: "The right to obtain communication...". This is an explicit link, but not recognizable by the system. The semantic link enables us to address this problem and to recover the exception in question.

\subsection{Query expansions based on ontoPrivacy}

An essential innovative feature of LMEmataSearch is the possibility to perform a conceptual searching based on ontoPrivacy.

Currently, the simple IS-A relations among ontoPrivacy classes do not permit a deep reasoning. Some experiments on query expansions based on our lightweight ontology are underway.

For example, if the user looks for the power regarding authorities the system response relies on the Role class 
defined within the domain ontology and it retrieves twenty paragraphs, where the addressee "Authorities" is spread into three different addresses: Garante, Judicial office and Higher Council of the Judiciary.

\section{RELATED WORKS}

In literature there are three main approaches to the representation and use of semantics of legal documents:

- Semantics of terms: modelling of semantics of the terms through their aggregations in concepts, classes and their relations (e.g. Berrueta et al., 2006).

- Semantics of terms and syntax of phrases: modelling of semantics of the terms through their aggregations in concepts, classes and their relations (as above), and their specialization based on their syntactic roles inside phrases (e.g. subject). (e.g. Debrauwer et al., 2008).

- Semantics of terms and sectorial knowledge models: modelling of semantics of the terms through their aggregations in concepts, classes and their relations (as above) and their specialization based on their illocutionary roles inside legal models.

The representation of contract cases for their conceptual retrieval (Dick, 1991), is based on the activity of lawyers in retrieving the information they need. A lexicon of legal concepts is used, while the principal argument of each case is analyzed according to Toulmin's argument model of analysis (Toulmin, 1969). Conceptual graphs are the notation used for the representations of conceptual relations. Our LME project belongs to this approach, being based both on general linguistic and sectorial legal models.

\section{CONCLUSIONS}

In this paper the LME project has been described in all its parts with particular attention to the design and the functionalities of its two modules. A formal provision theory, the ontoPrivacy lightweight ontology, the LMEmetaEdit tool for the drafting, editing and semantic mark-up of legal texts and many detailed examples of the LMEmetaSearch module have been presented.

Surface descriptions of a source result into defining documentary units, such as articles and paragraphs. The pragmatic and semantic contents description models, proposed in this paper, add virtual documentary meaningful units of substantive character to information systems: legislative rules and their argument contents.

The underlying idea of our project is to couple the traditional search for legal texts with the conceptual search by normative provisions and their contents.

The final version of the system will be required to yield units (articles, paragraphs, numbers, letters, or even sentences) including specific types of provision to be found in various laws at the same time. This should allow the easy identification of all the regulative contents provided for by all the laws regulating a given subject.

\section{REFERENCES}

Berrueta, D., Labra, J.E., Polo, L. (2006) 'Searching over Public Administration Legal Documents Using Ontologies', Frontiers in Artificial Intelligence and Applications, Vol. 140, pp.167175.

Biagioli, C. (1997) 'Towards a legal rules functional microontology'. Proceedings of LEGONT'97. Melbourne, Australia.

Biagioli, C. and Turchi, F. (2005) "Model and ontology based conceptual searching in legislative XML collections" LOAIT workshop, ICAIL 2005, Bologna Italy

Biagioli, C. and Grossi, D. (2008) 'Formal Aspects of Legislative Meta-Drafting'. To appear in Proceedings of Jurix08. Florence, Italy.

Breuker, J. and Hoekstra, R (2004) 'Epistemology and ontology in core ontologies: FOLaw and LRI-Core, two core ontologies for law'. Proceedings of EKAW Workshop on Core ontologies. Northamptonshire, UK.

Corcho, O., Fernández-López, M., Gómez-Pérez, A. (2003) 'Methodologies, tools and languages for building ontologies. Where is their meeting point?', Data \& Knowledge Engineering, Vol. 46, No. 1, pp.41-64.

Debrauwer, L., Karam, N., Brulé, J.P. (2008) 'Semantic Searching and Text Analysis with Distinguo ${ }^{\circledR}$ '. Obtained trought the internet: http://www.ultralingua.com/en/dox/Distinguo-WhitePaper.pdf, [accessed 09/10/2008].

Dick, J.P. (1991) 'Representation of legal text for conceptual retrieval'. Proceedings of the 3rd international conference on Artificial intelligence and law. Oxford, England.

Gangemi, A., Sagri, M., Tiscornia, D. (2005) 'Constructive framework for legal ontologies', In: Benjamins, V.R., Casanovas, P., Breuker, J., Gangemi, A. (eds.), Law and the Semantic Web, (pp.97-124), Berlin: Springer.

Hirst, G. (2003) 'Ontology and the lexicon', In: Staab, S., Studer, R. (eds.), Handbook on Ontologies in Information Systems, (pp.209-230), Berlin: Springer.

Honoré, A.M. (1977) 'Real laws', In: Hacker, P. M. S., Raz, J. (eds.), Law, Morality and Society Essays in Honour of H. L. A. Hart, (pp.99-118), Oxford: Oxford University Press.

Maat, E. de, Winkels, R. (2007) 'Categorisation of Norms'. Proceedings of Jurix 2007. Leiden, The Netherlands.

Rawls, J. (1955) 'Two Concepts of Rule', Philosophical Review, Vol. 64, pp.3-32.

Sartor, G. (2006) 'Fundamental Legal Concepts: A Formal and Teleological Characterisation, Artificial Intelligence and Law'. Technical report. European University Institute, Florence/Cirsfid, University of Bologna.

Shannon, C. E., Weaver, W. (1949) The Mathematical Theory of Communication, Urbana: University of Illinois Press.

Toulmin, S. (1969) The Uses of Argument, Cambridge: Cambridge University Press.

\section{WEBSITES}

Legal-RDF Vocabularies, at http://www.legal-rdf.org

Council Decision of 22 December 1998 concerning the Rules for Participation, at $\mathrm{ftp}: / / \mathrm{ftp} . c 0 r d i s . e u r o p a . e u / p u b / \mathrm{fp} 5 / \mathrm{docs} / \mathrm{en}-\mathrm{ec}-$ r.pdf

Decreto legislativo 30 giugno 2003, n. 196, at http://www.parlamento.it/parlam/leggi/deleghe/03196dl.htm

Law Making Environment project site: http://nir.ittig.cnr.it/lme 

